\title{
LA MIRADA DEL DOCENTE SOBRE LA PROMOCIÓN DE LA CONVIVENCIA ENTRE LAS FAMILIAS DE SU ESTUDIANTADO THE PERCEPTION OF TEACHER ABOUT THE PROMOTION OF COEXISTENCE WITHIN THE FAMILIES OF THEIR STUDENTS
}

\section{Volumen 14, Número 3 \\ Setiembre - Diciembre}

pp. 1-24

Este número se publicó el 30 de setiembre de 2014

Ana Rocío Barquero Brenes

Revista indizada en REDALYC, $\underline{\text { SCIELO }}$

Revista distribuida en las bases de datos:

CATÁLOGO DE LATINDEX, IRESIE, CLASE, DIALNET, DOAJ, E-REVIST@S, SHERPA/ROMEO, QUALIS, MIAR

Revista registrada en los directorios:

ULRICH'S, REDIE, RINACE, OEI, MAESTROTECA, PREAL, CLACSO 


\title{
LA MIRADA DEL DOCENTE SOBRE LA PROMOCIÓN DE LA CONVIVENCIA ENTRE LAS FAMILIAS DE SU ESTUDIANTADO \\ THE PERCEPTION OF TEACHER ABOUT THE PROMOTION OF COEXISTENCE WITHIN THE FAMILIES OF THEIR STUDENTS
}

\begin{abstract}
Ana Rocío Barquero Brenes ${ }^{1}$
Resumen: Se realizó un estudio de diseño mixto, con población docente de preescolar y primaria perteneciente a un centro educativo del Área Metropolitana en Costa Rica, durante los ciclos lectivos 2010 y 2011. Esto con el propósito de indagar las percepciones del personal en educación, sobre factores involucrados en la promoción de la convivencia entre las familias de su estudiantado. Asimismo, se les consultó sobre las estrategias que implementan en el aula para educar a favor del convivir. La recolección de información se llevó a cabo a través de cuestionarios, grupo focal y una escala tipo likert. Los resultados evidenciaron que el personal docente posee una visión amplia pero poco profunda del tema, su discurso no teoriza sino que se basa en ejemplos de experiencias cotidianas. Manifiestan experimentar una recarga en sus funciones educativas en tanto consideran que las familias han dejado de educar a niños y niñas en pro de la convivencia.
\end{abstract}

Palabras clave: CONVIVENCIA, DOCENCIA, FAMILIA, SOCIALIZACIÓN, CULTURA DE PAZ, COSTA RICA.

\begin{abstract}
This mix approach study was held with a subject group consisting of preschool and primary teachers from an educational center located in the Metropolitan Area of Costa Rica, between 2010 and 2011. The aim was to investigate the perceptions of the teachers about the factors that promote or inhibit the coexistence between the families of their students. Likewise, the strategies implemented at the classrooms to promote coexistence were consulted. The collection of information consisted of several questionnaires, a focus group, and a likert scale. Regarding the results, a wide but shallow perception of the topic could be evidence, as the staff discourse is not based on theory but it every day experiences. They seem to experiment an overload in their educating functions considering that the families are no longer educating their children to promote coexistence.
\end{abstract}

Key words: COEXISTENCE, TEACHING, FAMLILY, SOCIALIZATION, CULTURE OF PEACE, COSTA RICA.

\footnotetext{
1 Docente en la Escuela de Psicología de la Universidad de Costa Rica, también labora en un centro educativo. Máster en Psicología Educativa, Universidad de Costa Rica, San José, Costa Rica.
}

Dirección electrónica: ana.barquero@ucr.ac.cr

Artículo recibido: 21 de octubre, 2013

Devuelto para corrección: 6 de marzo, 2014

Aprobado: 15 de mayo, 2014 


\section{Introducción}

La formación para la convivencia ha sido tema central en los foros de la UNESCO y en los debates acerca de las dimensiones éticas de la sustentabilidad, de la defensa de los Derechos Humanos y de la integración en las sociedades multiculturales. A esta formación se han sumando, en las últimas décadas, diversos Ministerios de Educación Pública especialmente de países de habla hispana como Chile, Colombia, España y Costa Rica. Además, se han generado redes, políticas, planes y propuestas de intervención, para implementarse en instituciones de enseñanza pública y privada. Pero la promoción del convivir, desde el seno familiar y pese a su trascendental relevancia, ha sido poco explorada, aun menos si se estudia a través de la mirada del docente.

Es importante recordar que la familia, como primera instancia socializadora mediada por vínculos afectivos, se convierte en un escenario idóneo para enseñar a convivir. En su seno se desarrollan valores y competencias sociales como el descubrimiento de sí mismo y del otro, la empatía, la asertividad, la autonomía, el respeto, la tolerancia, el respeto a las diferencias, el sentido de justicia y la valoración de las diferencias, entre otras, todas como el cimiento del convivir. Sin embargo, el sistema escolar señala que en las últimas décadas ha surgido un debilitamiento de la familia como promotora de la convivencia y que esta función se ha recargado a la dinámica de la escuela.

Al respecto, López (2005) explica que las presiones sociales, el nivel de consumismo, la multiplicidad de funciones que deben asumir las personas a cargo de la crianza han disminuido la cantidad y la calidad del tiempo para compartir en familia, delegando el aprendizaje de la convivencia a la escuela. Partiendo de estos planteamientos se definieron los objetivos del presente estudio, dirigidos a conocer la percepción de cinco integrantes del equipo docente, de un centro educativo de enseñanza preescolar y primaria, acerca de los factores promotores e inhibidores de la convivencia entre las familias de su estudiantado. Asimismo, se contemplaron las sugerencias de estos profesionales de la educación a las familias. El último objetivo se dirigió a indagar acciones concretas que ejecuta el grupo docente, en el contexto escolar y en pro de la enseñanza de la convivencia. 


\section{Referente Teórico}

\subsection{Convivencia}

De acuerdo con el Ministerio de Educación de Chile (2008), la convivencia constituye la esencia de las relaciones sociales, es un aprendizaje que inicia en el espacio familiar para luego trasladarse a otros contextos. Este aprendizaje se encuentra ligado a la enseñanza de valores que constituyen el núcleo de una formación ciudadana democrática y en paz; requiere de la capacidad de las personas de vivir con otras en un marco de respeto y solidaridad, la posibilidad del reconocimiento y valoración de la diversidad, aceptando y apreciando otros puntos de vista, es decir

A convivir se aprende, no es algo que venga inscrito. La convivencia constituye la esencia de las relaciones sociales. El ser humano, por su naturaleza necesita de otros: es, se hace y construye en la relación con demás, tanto, que su existencia depende de la relación con sus semejantes. De allí la importancia capital de aprender a convivir. Este aprendizaje se logra en la casa, en el espacio familiar, en el barrio y también en el establecimiento educacional. (Ministerio de Educación Chile, 2008, p. 2)

En correspondencia con los planteamientos anteriores, para la política educativa colombiana (2005), la convivencia pacífica es una manera de vivir que exige aprender a practicarla puesto que es resultado del aprendizaje. Está involucrada con la capacidad de las personas para establecer relaciones sociales y humanas de calidad, fundamentadas en la tolerancia y en el respeto de los demás, por lo que se plantea que es necesario educar para cualificar las relaciones sociales y humanas, para vivir en armonía y para tramitar adecuadamente las desavenencias. Esto no solo desde referentes éticos, culturales y normativos, sino también desde competencias, habilidades y capacidades de los individuos para interactuar constructivamente.

La formación de sujetos habilitados para convivir armónicamente, además de ser un proceso de apropiación cognitiva de los valores de respeto a los derechos de los demás, de tolerancia, honestidad y solidaridad, es fundamentalmente un proceso de desarrollo de competencias que generen practicas proclives a la convivencia. Competencias como por ejemplo, la capacidad para manejar sentimientos y emociones, tramitar adecuadamente los conflictos, trabajar en grupo y compartir, 
buscar soluciones a los problemas, argumentar, dialogar y fijarse metas". (Ministerio de Educación de Colombia, 2005, p. 6)

Para la Unesco (2007), la manera en que se convive es cultural y es construida; además, contribuye a generar una vivencia de predictibilidad y seguridad, lo que no significa que no se presenten discrepancias, disensos y posiciones subversivas.

El convivir de personas en grupos e instituciones es un proceso constructivo donde ocurren transacciones, negociación de significados (Brunner, 1990), elaboración de soluciones, etc. Este convivir va creando un significado común construido históricamente (Geertz, 1994), de naturalidad y predictibilidad, que genera un sentido de familiaridad, un "así son las cosas y un así se hacen las cosas", que llega a formar parte de la identidad del grupo y de quienes participan en él. Es así como convivir en una u otra institución, supone convivir en el marco de una identidad de grupo, expresado en formas particulares de relación, lógicas de acción y significados, valoraciones y creencias instaladas. (UNESCO, 2008, p. 16)

A partir de estas propuestas sobre el proceso constructivo de la convivencia humana, adquiere relevancia revisar el papel del entorno familiar en cuanto agente primario promotor del convivir, así como lo que acontece en el espacio escolar.

\subsection{Convivencia escolar}

Retomando planteamientos de la política escolar chilena (Ministerio de Educación de Chile, 2002), la convivencia escolar es la particular relación que se produce en la comunidad educativa entre los diversos integrantes: estudiantes, docentes, directivos, asistentes de la educación, padres, madres y otras personas. En este espacio, la convivencia se enseña, se aprende y se refleja en los diversos contextos formativos y en los instrumentos de gestión.

Es un aprendizaje que se genera en la interrelación entre los diferentes miembros del establecimiento educacional y que tiene incidencia significativa en el desarrollo ético, socio-afectivo e intelectual del estudiantado. También, implica el reconocimiento y respeto por la diversidad, la capacidad de las personas de entenderse, de valorar y aceptar las diferencias y otros puntos de vista (Ministerio de Educación de Chile, 2002). 
La convivencia escolar es a la vez un desafío y un aprendizaje. Un aprendizaje, pues supone una enseñanza que está íntimamente ligada con el proceso educativo de la persona y, como tal, en directa relación con el contexto, el medio social y familiar donde se ha desarrollado. (Ministerio de Educación de Chile, 2008, p. 4 )

Por lo tanto, la educación para la convivencia supone un proceso de repensar la escuela. Esto demanda nuevos marcos para interpretar la realidad, diferentes actitudes y prácticas e implica la totalidad del centro y una coherencia entre el discurso y el funcionamiento real. Además, requiere de conciencia sobre la necesidad de cambiar formas de relación y comunicación, así como los estilos de poder y autoridad.

Para el Ministerio de Educación de Colombia (2005), como lugar de interacciones múltiples y variadas que se producen y se reproducen continuamente en todos los campos de la vida escolar, la escuela es un escenario idóneo para las prácticas de convivencia. Por lo tanto, proponen que la comunidad educativa en el mundo de hoy debe tener por norte enseñar a vivir juntos en armonía y a convivir con base en el respeto a las diferencias y a los derechos de los demás, otorgándole a esa institución una responsabilidad ineludible en la formación de ciudadanos capaces de ejercer la democracia, respetar los derechos humanos y relacionarse entre sí de manera constructiva.

De acuerdo con Muñoz, Saavedra y Villalta (2007), el tema de la convivencia escolar toma cada vez más relevancia en las comunidades educativas, enfocadas no solo en intervenir, sino también en prevenir situaciones de violencia escolar. Los medios de comunicación y las investigaciones muestran día a día la situación de tensión cotidiana que emerge en los contextos educativos de diversas culturas a nivel mundial, que evidencia la complejidad creciente de las escuelas, las cuales se transforman en espacios representativos de los modelos sociales que influyen tanto de manera positiva como de forma negativa en el desarrollo y aprendizaje de los estudiantes.

En la misma línea de pensamiento, para la Unesco (2008) la educación para la convivencia, que implica la democracia y la paz, supone la necesidad de construir intencionalmente un ambiente educativo pertinente y relevante que eduque para la democracia y el convivir. Por lo tanto, en la experiencia escolar formativa se deben desarrollar valores, actitudes y habilidades socio-emocionales y éticas que sustenten una 
convivencia social donde se integre a todos y todas, para participar, compartir y desarrollarse plenamente.

Sin embargo, es importante no olvidar que la labor del equipo escolar tiene sus cimientos en la formación recibida en el núcleo de formación primaria: la familia, ente social en que inicia la inducción al convivir a través de los vínculos afectivos que se establecen.

\subsection{Familia como promotora del convivir}

Para Villarroel y Sánchez (2002), la familia es el primer grupo referencial con que cuenta el individuo, la instancia social que introduce en las relaciones íntimas y personales y proporciona las primeras experiencias; por tanto, el agente socializador más importante durante toda la niñez. Las intensivas experiencias sociales que ocurren en su seno, indican las autoras, son la base de la personalidad, independientemente de los cambios que experimenten más tarde en la vida como adolescentes o como adultos.

De acuerdo con Rodríguez (2006), la familia juega un papel protagónico respecto de la creación de ambientes que permitan establecer relaciones no violentas y, por lo tanto, aprender sobre la solución pacífica de conflictos. Destaca Rodríguez la importancia de reproducir patrones de respeto y tolerancia entre sus miembros, a fin de que estas habilidades se multipliquen en el intercambio social con otros sistemas.

En este sentido, Villarroel y Sánchez (2002) refieren que la familia en tanto instancia socializadora primaria, introduce a los nuevos miembros de la sociedad en el aprendizaje de las diversas normas, pautas y valores que a futuro les permitirán vivir autónomamente en sociedad. Sin embargo, resaltan que este asignado social ha venido en detrimento, y otros agentes sociales como la escuela han asumido muchas de sus funciones.

López (2005) refuerza esta idea, afirmando que en las últimas décadas la familia ha delegado el aprendizaje de la convivencia a la escuela indicando como la principal causa el poco tiempo con que cuentan los encargados de la crianza para involucrarse en las actividades y enseñanzas de niños y niñas.

Al respecto, Espinoza (2009) explica la situación a partir de las modificaciones que ha venido experimentando el grupo familiar, en especial, el papel protagónico de los factores económicos, que ha demandado adecuaciones y afectaciones importantes a la dinámica familiar; en conclusión, señala Espinoza, las familias se encuentran ante retos y 
cambios estructurales que invariablemente repercuten y repercutirán en las relaciones psico-emocionales.

\section{Metodología}

La investigación se llevó a cabo entre los años 2010 y 2011, en una institución educativa privada, con una población promedio de 160 estudiantes de los niveles correspondientes a preescolar y primaria, ubicada en una provincia del Área Metropolitana en Costa Rica. La población de estudio fue un subgrupo del personal docente, con quienes se indagó sobre las percepciones que poseen en relación a factores involucrados en la promoción de la convivencia entre las familias de su estudiantado. Asimismo, a este grupo participante se le consultó sobre las estrategias que implementan en el aula para educar a favor del convivir

En términos generales, el estudio se desarrolló en tres fases: la primera consistió en la revisión bibliográfica, que posibilitó la recopilación de información relevante y necesaria para estudiar el problema en cuestión y permitió elaborar el diseño de la investigación. En la segunda fase se procedió a la selección de la población con base en criterios de inclusión previamente establecidos, para continuar con las técnicas de recolección de la información que posibilitaron la obtención de datos cualitativos y cuantitativos. Finalmente, en la tercera etapa tuvo lugar el procesamiento y análisis de la información.

\subsection{Tipo de estudio y diseño de investigación}

En los antecedentes estudiados fue poca la documentación sobre investigaciones realizadas en el contexto escolar costarricense vinculadas con la percepción del personal docente sobre la convivencia familiar. Por lo tanto, se consideró pertinente realizar un planteamiento exploratorio. La aproximación exploratoria, de acuerdo con Hernández, Fernández y Baptista (2006), además de utilizarse para recolectar información sobre temáticas poco estudiadas, pretende realizar indagaciones desde perspectivas novedosas, buscando desplegar datos que faciliten la profundización posterior en el tema. Asimismo, estos estudios permiten realizar un análisis más integral de un contexto particular.

Adicionalmente, la investigación se realizó desde un enfoque mixto que, de acuerdo con Hernández, Fernández y Baptista (2006), se caracteriza por recolectar, analizar y vincular datos cuantitativos y cualitativos en un mismo estudio; por lo tanto, las técnicas y 
aproximaciones metodológicas que se utilizaron responden a ambos enfoques de investigación. La riqueza de este diseño mixto permitió explorar y ampliar desde diversas perspectivas el problema de estudio, comprendiéndolo más integralmente.

\subsection{Población de estudio}

El equipo docente de la institución seleccionada, en el momento del estudio, se encontraba constituido por 19 personas, distribuidas por niveles y materias: cuatro de ellas impartían lecciones en la sección de preescolar, nueve personas en enseñanza primaria y las seis restantes eran profesionales de materias especiales. Asimismo, del total del personal docente un $90 \%$ correspondió a población femenina y un $10 \%$ masculina.

La selección del grupo participante se definió a partir del cumplimiento de los siguientes criterios de inclusión: poseer estudios universitarios acordes con el puesto desempeñado, impartir lecciones a niños y niñas entre los tres y los diez años de edad, contar con un mínimo de cinco años de labor dedicada a la docencia y al menos tres años de servicio en la institución.

Estos criterios preseleccionaron siete participantes, dos de ellas fueron asignadas como grupo piloto y cinco como participantes en el estudio. El paso siguiente fue firmar un consentimiento informado, que fue presentado de forma oral y escrita, y que explicó los objetivos de la investigación, riesgos y beneficios posibles, la duración y participación voluntaria, el retiro voluntario y el manejo de la confidencialidad.

En la Tabla 1, se detallan datos personales y laborales de las cinco docentes seleccionados en el estudio.

\begin{tabular}{lrlccl}
\hline \multicolumn{5}{c}{ Datos personales y laborales de las docentes } \\
\hline Docente & Sexo & Edad & $\begin{array}{c}\text { Años trabajo } \\
\text { en docencia }\end{array}$ & $\begin{array}{c}\text { Años laborar para } \\
\text { el Centro Educativo. }\end{array}$ & Niveles que imparte \\
\hline & & & 16 & I y II \\
1 & Femenino & 66 & 23 & 12 & I, II y III \\
2 & Femenino & 60 & 13 & 17 & Preescolar \\
3 & Femenino & 42 & 23 & 3 & III y IV \\
4 & Femenino & 30 & 8 & 5 & I, III, IV y V \\
5 & Femenino & 50 & 25 & & \\
\hline
\end{tabular}


Los datos refieren que el equipo participante fue en su totalidad femenino, con edades variables entre los 30 y los 66 años, para un promedio de 50 años de edad. Con respecto al tiempo de servicio como docente, osciló entre los 8 y los 25 años para un promedio de 18 años, lo que indica un nivel de experiencia alto. Adicionalmente, se sitúo en 10 años el promedio de tiempo laborado en la institución. Es importante mencionar que la totalidad de las participantes, tal y como se solicitó, contaba con estudios universitarios acordes al puesto que desempeñaba.

\subsection{Técnicas de Recolección de la Información}

Las técnicas de recolección del presente estudio consistieron en la ejecución de un grupo focal directivo y la aplicación de tres instrumentos que permitieran obtener información cuantitativa y cualitativa acerca del tema. Previamente a la aplicación de los instrumentos al grupo participante, se contó con la retroalimentación de un grupo piloto (conformado por dos maestras), que posibilitó la reestructuración de los implementos originales.

Luego, se procedió a la administración en cuatro etapas sucesivas. En primera instancia se suministró un cuestionario semi estructurado, dirigido a indagar la percepción de las docentes acerca de factores promotores e inhibidores de la convivencia entre las familias del estudiantado. Adicionalmente, se les solicitó brindar sugerencias que, a juicio de las participantes, incentivara el valor del convivir en el núcleo familiar.

La segunda etapa consistió en la participación de las docentes en un grupo focal directivo, lo que permitió profundizar en la información previamente obtenida a través del cuestionario. En la discusión se incentivó la interacción discursiva y la contrastación de las opiniones entre las participantes. La experiencia se registró mediante el uso de cámara de video, para luego transcribirla y sistematizarla en categorías de análisis.

En un tercer momento se aplicó una escala tipo likert, donde las participantes asignaron un nivel de importancia a 22 factores, relacionados con la temática del estudio.

En la cuarta etapa se dispuso de un segundo cuestionario semi estructurado, dirigido a indagar acciones concretas realizadas por las maestras en la dinámica escolar, en pro de la enseñanza de la convivencia.

Una vez finalizada la fase de recolección de información, se procedió a sistematizar datos cuantitativos y cualitativos más relevantes y, a partir de estos, derivar cuatro categorías de análisis que se explican a continuación. 


\section{Análisis de resultados}

Los resultados cualitativos y cuantitativos, obtenidos a través de los diferentes instrumentos de medición, se sistematizaron en las siguientes categorías de análisis:

a) Percepción del equipo docente sobre los factores que promueven la convivencia entre las familias de sus estudiantes.

b) Percepción del equipo docente sobre los factores que inhiben la convivencia entre las familias de sus estudiantes.

c) Sugerencias del equipo docente a las familias de sus estudiantes en pro de la enseñanza de la convivencia.

d) Percepción del equipo docente sobre el convivir y acciones que realizan en el aula para su promoción.

\subsection{Percepción del equipo docente sobre los factores que promueven la convivencia entre las familias de sus estudiantes}

La totalidad de las docentes afirmó que la familia es el espacio social donde se debe iniciar el aprendizaje de valores, hábitos, costumbres, un estilo comunicacional, formas de compartir y de relacionarse y, por lo tanto, se aprenden habilidades sociales para la vida. Acerca de los factores promotores del convivir en el seno familiar identificaron como relevantes los siguientes: la vivencia de valores, el tiempo para compartir en cantidad y calidad, la aplicación de métodos disciplinarios y la enseñanza de normas sociales, los mismos se explicitan, junto a los ejemplos más significativos en la Tabla 2. 


\section{TABLA 2}

Percepción de las docentes sobre los factores promotores de la convivencia en las familias de sus estudiantes.

\begin{tabular}{|c|c|c|}
\hline $\begin{array}{c}\text { Factores } \\
\text { promotores: }\end{array}$ & $\begin{array}{l}\text { Síntesis de aspectos } \\
\text { relevantes de las } \\
\text { argumentaciones: }\end{array}$ & $\begin{array}{l}\text { Ejemplos más significativos brindados por el } \\
\text { grupo: }\end{array}$ \\
\hline $\begin{array}{l}\text { vencia } \\
\text { lores }\end{array}$ & $\begin{array}{l}\text { Practicar en familia valores } \\
\text { como el amor, la tolerancia, } \\
\text { la solidaridad, la } \\
\text { responsabilidad y el } \\
\text { respeto. Buscar } \quad \text { el } \\
\text { bienestar de los demás y } \\
\text { no solo el propio. Aprender } \\
\text { a relacionarse sin } \\
\text { rechazos, burlas } \quad \text { o } \\
\text { discriminaciones. }\end{array}$ & $\begin{array}{l}\text { "...si en una familia se tratan con respeto, eso se } \\
\text { nota demasiado, el chiquito lo refleja con su conducta } \\
\text { y aprende" } \\
\text { "...hay alumnos a los que si les enseñan a } \\
\text { preocuparse por los otros, entonces es bonito porque } \\
\text { ellos integran y son solidarios" } \\
\text { "...el niño que es responsable es porque aprendió en } \\
\text { la casa y así sigue en la escuela y después en la } \\
\text { vida". }\end{array}$ \\
\hline $\begin{array}{lr}\text { Compartir } & \text { en } \\
\text { calidad } & y \\
\text { cantidad } & \end{array}$ & $\begin{array}{l}\text { Personas adultas que } \\
\text { dediquen tiempo en calidad } \\
\text { y cantidad. No es suficiente } \\
\text { con llamadas telefónicas o } \\
\text { el uso de otros medios } \\
\text { electrónicos para } \\
\text { monitorear a los niños y } \\
\text { niñas. }\end{array}$ & $\begin{array}{l}\text { "...hay papás que sí sacan el tiempo para estar con } \\
\text { los hijos, hasta piden permiso en el trabajo para venir } \\
\text { a un acto cívico y apoyarlo". } \\
\text { "...es que hay que tener tiempo para leer cuentos, } \\
\text { para jugar, conversar, hacer tareas, sino mejor no } \\
\text { comprometerse a ser padre”. } \\
\text { "...hay mamás que no les interesa el hijo, no hablan } \\
\text { con él, no juegan, no enseñan, lo dejan ver tele toda } \\
\text { la tarde para que no las moleste. Entonces, ¿de qué } \\
\text { sirve estar con él?". }\end{array}$ \\
\hline os & $\begin{array}{l}\text { Emplear una combinación } \\
\text { de amor y disciplina, en el } \\
\text { que no se necesite recurrir } \\
\text { a los golpes pero si a la } \\
\text { firmeza y aplicar } \\
\text { consecuencias. }\end{array}$ & $\begin{array}{l}\text { "...hay familias como la de } x x \text { dónde hay amor con } \\
\text { rigor y vean a la chiquita, una excelente niña y } \\
\text { compañera" } \\
\text { "...se requieren papás y mamás que si quieran } \\
\text { educar, que no se contradigan y que pongan límites } \\
\text { sin miedo, de esa forma si van a enseñar } \\
\text { convivencia". }\end{array}$ \\
\hline $\begin{array}{l}\text { Ense } \\
\text { norm } \\
\text { socia }\end{array}$ & $\begin{array}{l}\text { Enseñar normas de } \\
\text { cortesía que se relacionen } \\
\text { con el respeto y la } \\
\text { búsqueda del interés y } \\
\text { bienestar de los otros. }\end{array}$ & $\begin{array}{l}\text { ortante enseñarles desde chiquitos normas } \\
\text { a, como decir con permiso, o pedir por } \\
\text { lo hace a uno respetuoso de los demás, un } \\
\text { adano". }\end{array}$ \\
\hline
\end{tabular}

En el discurso del grupo del estudio se dimensionó la promoción de la convivencia en la familia, como una serie de enseñanzas que se transmiten en la interacción directa en cantidad y calidad con los niños y las niñas. Por tanto, se partió de la creencia de que las conductas adultas modelan formas de relación que son imitadas por la población infantil, y que, posteriormente, se reproducen en otras esferas sociales como la escuela. A su vez, la aplicación de métodos disciplinarios efectivos viene a ser para las participantes, la forma de regular las conductas dirigidas al convivir de las que no lo son.

Esta serie de argumentaciones sobre los factores contempló varias de las dimensiones a las que hacen alusión ministerios de educación de gobiernos 
latinoamericanos, como Chile 2008 y Colombia 2005, donde se enfatiza en la convivencia como un proceso que se enseña y se aprende, y que se deriva de la trascendencia de fortalecer valores como la tolerancia, el respeto, la solidaridad y la empatía.

Es interesante considerar que los factores citados por las profesoras se ubican plenamente en el plano de lo conductual, y que otros componentes de índole afectiva o cognitiva no son valorados por ellas. Por ejemplo, se deja de lado que la convivencia sea un proceso de apropiación cognitiva, acorde con un estilo de vida que origina pensamientos y sentimientos que, finalmente, se expresan a través de conductas (Ministerio de Educación de Chile, 2002). Más bien, se antepone el tema conductual, esperando también resultados inmediatos a este nivel, sin tenerse en la trascendencia de lo cognitivo ni de lo afectivo.

A fin de profundizar en los datos anteriores, al grupo se le presentó una lista de 21 enunciados que incluían conductas, valores, y aspectos emocionales. A las maestras se les solicitó otorgar un nivel de importancia (entre los rangos de alta importancia a ninguna importancia), tomando como referente la trascendencia de los mismos como prácticas proclives al convivir. Los resultados se detallan en la Tabla 3.

\begin{tabular}{lcccc}
\hline \multicolumn{5}{c}{ Grado de importancia, que el grupo de docentes, le atribuye a variables en relación a la } \\
& promoción de la convivencia familiar & \\
\hline & Alta & Media & Baja & Ninguna \\
\hline Variables & Importancia & importancia & importancia & importancia \\
\hline Hábitos & $100 \%$ & 0 & 0 & 0 \\
Límites & $100 \%$ & 0 & 0 & 0 \\
Consecuencias & $100 \%$ & 0 & 0 & 0 \\
Empatía & $100 \%$ & 0 & 0 & 0 \\
Asertividad & $100 \%$ & 0 & 0 & 0 \\
Resolución conflictos & $100 \%$ & 0 & 0 & 0 \\
Autonomía & $100 \%$ & 0 & 0 & 0 \\
Participar labores hogar & $100 \%$ & 0 & 0 & 0 \\
Compartir tiempo & $100 \%$ & 0 & 0 & 0 \\
Solidaridad & $100 \%$ & 0 & 0 & 0 \\
Responsabilidad & $100 \%$ & 0 & 0 & 0 \\
Honestidad & $100 \%$ & 0 & 0 & 0 \\
Tolerancia & $100 \%$ & 0 & 0 & 0 \\
Ver programas y películas & $60 \%$ & $20 \%$ & $20 \%$ & 0 \\
Dar el ejemplo & $100 \%$ & 0 & 0 & 0 \\
Afecto físico & $80 \%$ & $20 \%$ & 0 & 0 \\
Afecto a través de palabras & $100 \%$ & 0 & 0 & 0 \\
Apoyo estudios & $100 \%$ & 0 & 0 & 0 \\
Respeto puntos de vista & $100 \%$ & 0 & 0 & 0 \\
Acuerdos y compromisos & $100 \%$ & 0 & 0 & 0 \\
Compartir & $100 \%$ & 0 & 0 & \\
\hline
\end{tabular}


Los datos proporcionados por la escala likert fueron uniformes, en otras palabras, se orientaron en una misma dirección. El valor de mayor frecuencia, o el que se repitió más veces (moda), fue el que correspondió a "alta importancia". Las variables 'ver programas y películas en familia' y 'la expresión de afecto a través de manifestaciones físicas' fueron consideradas por dos docentes de mediana importancia, mientras que para el resto estuvieron un $100 \%$ asociadas con la promoción de la convivencia.

En síntesis, para el equipo de maestras, la enseñanza de hábitos, la puesta de límites, la aplicación de consecuencias, enseñar a los niños y niñas a comunicarse con asertividad, la empatía, enseñar formas alternas de resolver conflictos, la autonomía, colaborar con labores hogareñas, el aprendizaje a través del ejemplo, y la vivencia de valores (solidaridad, responsabilidad, honestidad, tolerancia, respeto, compartir y gratitud) son factores de alta importancia en el tema de la promoción de la convivencia en el seno familiar, lo que coincide a plenitud con los cuatro factores que identificaron como promotores, con restar importancia al plano afectivo, por lo que refiere consistencia en sus planteamientos.

\subsection{Percepción del equipo docente sobre los factores que inhiben la convivencia entre las familias de sus estudiantes}

Al consultar al equipo participante, su percepción sobre los factores que inhiben la convivencia entre las familias de sus estudiantes, se obtuvieron los siguientes datos que se sistematizan en la Tabla 4. 


\begin{tabular}{|c|c|}
\hline \multicolumn{2}{|c|}{$\begin{array}{c}\text { TABLA } 4 \\
\text { Percepción de las docentes sobre los factores que inhiben la convivencia en las familias de } \\
\text { sus estudiantes }\end{array}$} \\
\hline $\begin{array}{l}\text { Factores que } \\
\text { inhiben }\end{array}$ & Ejemplos más significativos proporcionados por el grupo \\
\hline $\begin{array}{l}\text { Enseñanza de } \\
\text { antivalores }\end{array}$ & $\begin{array}{l}\text { “...ahora hay mucho chiquito egoísta que no quiere compartir ni ser solidario, solo } \\
\text { piensa en sí mismo". } \\
\text { "...a los niños no se les educa para ser solidarios, para preocuparse por los } \\
\text { hermanos o los papás, se les enseña a ser egoístas y por eso aquí en la escuela } \\
\text { pelean y no comparten". } \\
\text { "... hay chiquitos que disfrutan presumiendo con otros de lo que tienen". } \\
\text { "... no los enseñan a valorar, los chiquillos creen que las cosas caen del cielo y que } \\
\text { "...los alumnos de los papás comprarles celular y todo". } \\
\text { tienen tolerancia". } \\
\text { "... hay muchos de autocontrol, o es muy pobre, no aguantan nada, no } \\
\text { quiere que uno haga trampa para que el hijo gane". }\end{array}$ \\
\hline $\begin{array}{l}\text { Tiempo limitado } \\
\text { para compartir }\end{array}$ & $\begin{array}{l}\text { "... hay papás demasiado perdidos, nunca saben nada de lo que pasa con el hijo, } \\
\text { sabemos más nosotras". } \\
\text { "...en mi grupo hay varios chiquitos que solo ven a los papás en la mañana un } \\
\text { ratito antes de venir a la escuela y eso es todo". } \\
\text { "...para que el chiquillo pase entretenido y no los moleste, les compran de todo y } \\
\text { no les enseñan a valorar". } \\
\text { "... los enseñan a que por todo hay premio, a ser insensibles y superficiales". } \\
\text { "...antes los papás se preocupaban por los hijos y por enseñarles para la vida, } \\
\text { ahora no gastan tiempo en eso y piensan que lo que hay que hacer es pagarles } \\
\text { una escuela privada para que sean bilingües y después estén bien } \\
\text { económicamente". } \\
\text { "...no los regañan para que los chiquillos no se enojen con ellos ni les reclamen, } \\
\text { porque se sienten culpables". } \\
\text { "...parece que ahora los padres no quieren educar a los hijos y esperan que aquí } \\
\text { en la escuela los eduquemos, hay chiquitos que ni siquiera saben saludar". }\end{array}$ \\
\hline $\begin{array}{l}\text { Falta de claridad } \\
\text { acerca de reglas } \\
\text { y figuras } \\
\text { autoridad }\end{array}$ & $\begin{array}{l}\text { Al respecto refirieron los siguientes ejemplos: “...en las casas los papás solo } \\
\text { amenazan pero no cumplen y los chiquillos lo saben”. } \\
\text { "...además si hay un problema y el chiquito es responsable, el papá viene aquí a la } \\
\text { escuela a defenderlo y nos amenaza a nosotras". } \\
\text { "...o sea los papás no aplican consecuencias y no dejan que nosotros las } \\
\text { apliquemos y después se quejan de que no les hacen caso. ¿Cómo van aprender } \\
\text { convivencia?". "...los papás los pasan salvando y no dejan que asuman sus } \\
\text { responsabilidades". }\end{array}$ \\
\hline $\begin{array}{l}\text { Estilos de } \\
\text { crianza }\end{array}$ & $\begin{array}{l}\text { “...( los padres) dicen una cosa pero hacen otra, hablan con uno de la honestidad } \\
\text { y que le enseñan al hijo a ser sincero, pero si hay un problema lo entrenan para } \\
\text { mentir y engañar para que salga librado"..... muchos papás están perdidos y no } \\
\text { saben cómo educar a los propios hijos, antes no pasaba eso, por eso es que no } \\
\text { hay convivencia”. } \\
\text { "...unos son todos despreocupados y no se enteran de las necesidades del } \\
\text { chiquito y otros son sobre involucrados y creen que ellos están matriculados en la } \\
\text { escuela". } \\
\text { "...surgen discusiones y problemas en la familia y los papás no entienden que ellos } \\
\text { tienen la culpa, le echan la culpa a la escuela, a la maestra que pone tanta tarea, } \\
\text { pero ellos no le han enseñado a los hijos a estudiar en casa, unos porque les da } \\
\text { pereza, otros porque les hacen todo". }\end{array}$ \\
\hline $\begin{array}{l}\text { Resolución } \\
\text { conflictos a } \\
\text { través de la } \\
\text { violencia }\end{array}$ & $\begin{array}{l}\text { "...algunas veces los padres le cuentan a uno que no pueden con el hijo, quieren } \\
\text { consejo, porque sienten como que lo matan, y algunos en realidad los golpean } \\
\text { porque no saben otra forma de conseguir obediencia". }\end{array}$ \\
\hline
\end{tabular}


Entre las participantes fue muy destacado el tema de los antivalores como un inhibidor de la convivencia, consideraron que algunas familias practican y enseñan la deshonestidad, el irrespeto, la intolerancia, el egoísmo, el desamor y la indiferencia, en otras palabras, dictan y modelan pautas morales incongruentes y contrarias al convivir. De acuerdo con ellas, esto sucede debido a que promueven el tener antes que el ser, optando por un estilo de vida materialista, que potencia comportamientos que calificaron de egoístas y contrarios a la convivencia, bajo esas circunstancias los niños y niñas aprenden a recibir, pero no a dar. Es importante destacar que las profesoras relegan la responsabilidad de manera directa al grupo social primario, sin considerar, como lo plantea Tuvilla (2004) que la instancia socializadora familiar realiza elecciones sobre cómo desempeñar su práctica, que no son azarosas ni arbitrarias, sino que obedecen a componentes culturales, políticos y económicos, que privilegian modelos de crianza específicos. Para Maturana (2007), los intereses políticos orientan hacia el consumismo, donde se adquiere importancia de acuerdo a los bienes que se poseen. El desarrollo de habilidades sociales no es importante, por lo que se crece inhabilitado en las mismas.

En relación con el punto anterior, las profesionales en educación coincidieron en que otra dificultad para promover la convivencia en la familia, se debe a que los padres y madres cuentan con poco tiempo en cantidad y calidad y esto interviene negativamente en la labor socializadora. De acuerdo con ellas, esta carencia responde a la multiplicidad de funciones que se deben a desempeñar simultáneamente, por lo que la crianza se relega y se limita a un monitoreo de conductas, que llamaron: educar por control remoto. Al respecto, comentaron que esto no es efectivo y que sus estudiantes se quejan del poco tiempo de que disponen las figuras de crianza para atender sus necesidades, que en muchos casos se sienten sustitutas de padres y madres.

En concordancia con lo expuesto, Espinosa (2009) señala que las familias actuales, con niños y niñas en edad preescolar y primaria, experimentan grandes demandas y presiones económicas que obligan al padre y la madre a asumir largas jornadas laborales. En el mismo sentido, Murueta (2009) contempla que la familia actual cuenta con poco tiempo para comunicarse, compartir y divertirse, y que debido al cansancio y a las limitaciones económicas son pocas los que aprovechan para promover la convivencia activa.

El grupo del estudió coincidió en que el poco tiempo dedicado a compartir genera sentimientos de culpabilidad en las personas adultas, que luego les impide aplicar 
consecuencias oportunas. Refieren que compensan la carencia con exceso de tolerancia, falta de límites, o mediante obsequios materiales en especial tecnológicos.

Para ellas, esto explica por qué en muchas de las familias de su estudiantado, no esté claro quiénes son las figuras de autoridad, situación que se traslada al contexto del aula, donde las docentes no son percibidas como personas a las que deben respetar y de quienes seguir instrucciones.

Para la investigadora, Espinosa (2009), en la actualidad las figuras parentales se sienten incompetentes en el establecimiento firme de reglas, debido a que temen ser vistos como malos padres si ejercen la autoridad. Asimismo, afloran sentimientos de culpabilidad por dejar a los hijos solos, debido a las actividades laborales o por haber vivido una separación, lo que condiciona a una jerarquía ineficaz o se produce una relación amigos-padres, donde los hijos no tienen una guía y deben ser más bien un soporte emocional de sus propios progenitores

Otro factor mencionado por las maestras como inhibidor de la convivencia, y ligado al punto anterior, se enfocó en los estilos de crianza inconsistentes, o cuando se cae en los extremos de la sobreprotección o el autoritarismo. Para ellas, estos estilos impiden la enseñanza y/o reforzamiento de habilidades sociales y valores, lo cual se traslapa al contexto educativo creando un clima de tensión y de discordia entre estudiantes, que dificulta la práctica de la convivencia

Por último, se refirieron a los riegos de la resolución de conflictos a través de la violencia. Para la población participante, muchas de las personas adultas ante situaciones de conflicto modelan a los niños y niñas actitudes de indiferencia, rechazo, venganza, y discriminación; algunas recurren a chantajes a través de premios y otras, por el contrario, pretenden resolver los conflictos a través del empleo de gritos, golpes e insultos; todos escenarios donde se excluye la comunicación asertiva.

Para Edwards (2002) en nuestra cultura no se enseña a resolver conflictos con asertividad, sino que existe un sistema de creencias que los estigmatiza como negativos y a las personas que los explicitan, como conflictivas. Esto ha generado actitudes de evasión, temor y desconfianza, que tienden a atribuir a los desacuerdos características negativas y amenazadoras. En este contexto, se desaprovecha la oportunidad para aprenden a resolver desavenencias, practicar la comunicación asertiva, la escucha, la posibilidad de ponerse en el lugar del otro, la creatividad para resolver situaciones complejas y estrategias de negociación. 
En síntesis, el equipo participante en el estudio concluyó que la familia ya no es la base de la sociedad, en tanto que las funciones socializadoras que le fueron encomendadas, se le reasignaron a la instancia escolar. Situación que valoraron como injusta, porque recarga sus funciones, las agobia, y las convierte en lo que llamaron: maestras-madres.

En cuanto a la responsabilidad de esta recarga de funciones, las profesoras la dirigen al núcleo familiar, percibido como negligente en el cumplimiento de sus tareas. Por tanto, no dimensionan el contexto social en que se encuentra inmerso. De acuerdo con Ares (2002), la familia se ha diversificado con el paso del tiempo, dado que es una categoría histórica, determinada por el sistema social imperante, que condiciona estilos de vida, jerarquías y funciones.

\subsection{Sugerencias del equipo docente a las familias de sus estudiantes en pro de la enseñanza de la convivencia}

La totalidad de profesoras consideró que el núcleo familiar debe fortalecerse si se desea aprender a convivir pacíficamente. Porcentualmente, un $60 \%$ opinó que la familia no prepara a los niños y niñas en el aprendizaje de la convivencia. El restante $40 \%$ señaló que no se puede generalizar, que algunas familias sí realizan acciones de apoyo, dedican tiempo a la crianza, se preocupan por promover disciplina e instaurar valores, dan amor y logran un ambiente familiar de tranquilidad y paz. Los datos se observan en el Gráfico 1.

\section{Gráfico \#1 \\ Percepción de docentes sobre la preparación que hacen las familias de sus integrantes para la cultura de paz.}

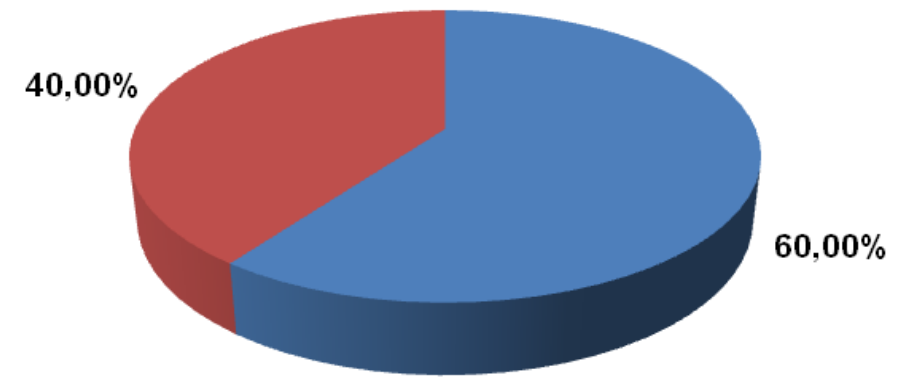

- Familia no preparan para cultura de paz

- Familia si preparan para cultura de paz 
El grupo de estudio se encuentra dividido al tener que considerar si la preparación para el convivir tiene o no lugar en el contexto familiar, siendo mayor (60\%) el subgrupo que considera que no se da, lo que coincide con la percepción de las docentes sobre los cambios que deben darse en la estructura familiar.

De forma paralela, se solicitó a las profesoras participantes proponer temas que consideraran relevantes para la capacitación de familias a fin de hacer proclive la práctica de la convivencia, temas que se enlistan con sus respectivos porcentajes en la Tabla 5.

\begin{tabular}{lc}
\hline \multicolumn{2}{c}{ TABLA 5 } \\
Preferencias temáticas propuestas por equipo docente para la \\
capacitación de familias
\end{tabular}

En lo que respecta a los temas sugeridos hubo una total concordancia con lo señalado en cuanto a los factores promotores e inhibidores. Prevaleciendo el tema de los valores y el de la disciplina. En cuanto al primero, con un porcentaje del 40\%, destacaron capacitar a las personas adultas para que instauren valores que calificaron como cimientos del convivir (compañerismo, solidaridad, compartir, preocupación por el otro y los valores cristianos).

En un $33 \%$ se refirieron a la importancia de instruir en la puesta de límites y aplicación de consecuencias; así como transmitir la relevancia de enseñar a los niños y niñas a seguir reglamentos y respetar figuras de autoridad. Una persona destacó la capacitación en temas de inteligencia emocional, explicitando el aprender a comunicarse, la empatía y el control de las emociones. Por último, la cultura de paz se relacionó, directamente con la convivencia, en tanto se consideró que las personas encargadas de la crianza desconocen o no se percatan de que educar para la convivencia es construir cultura de paz y que esto debe enseñarse.

Es interesante considerar que la selección de estas temáticas, por parte de las profesoras, se enfocó más en alternativas para facilitar su labor docente, en términos de que las enseñanzas del contexto familiar se trasladan posteriormente a la escuela. $Y$ no 
de una visión del convivir como un compromiso colectivo que implica un proyecto social, un replantearse la humanidad que queremos.

Además de las temáticas de capacitación, se les solicitó a las participantes brindar sugerencias a las familias a fin de vivir y enseñar convivencia. Los resultados se exponen en la Tabla 6.

\begin{tabular}{ll}
\hline \multicolumn{2}{c}{$\begin{array}{c}\text { TABLA 6 } \\
\text { Sugerencias que realizan docentes a familias para promover la } \\
\text { convivencia }\end{array}$} \\
\hline \multicolumn{1}{c}{ a familias } & $\%$ \\
\hline $\begin{array}{l}\text { Sugerencias } \\
\text { actividades y juegos. }\end{array}$ & $60 \%$ \\
Vivencia en valores & $20 \%$ \\
Fortalecer la comunicación & $20 \%$ \\
\hline TOTAL & $100 \%$ \\
\hline
\end{tabular}

Las docentes plantearon tres sugerencias como prácticas proclives al convivir, las mismas coincidieron a plenitud con los temas de capacitación y con los factores promotores. El componente relacionado con dedicar tiempo en calidad y cantidad que permita estar juntos, brindar atención, realizar actividades y juegos encabezó la lista con un porcentaje de respuesta del 60\%. Esto indica que, para el profesorado, gran parte de la solución está relacionada con pasar más tiempo compartiendo, lo cual puede resultar engañoso, debido a que de ser esto posible, podría no estar necesariamente enlazado a un estilo de vida que tenga por norte la cultura de paz.

Además, resulta poco realizable ante demandas sociales crecientes que orientan hacia tiempos cada vez más cortos de convivencia. Con porcentajes de $20 \%$ cada uno, el equipo participante sugirió a las familias la vivencia más que el discurso verbal de valores, y el fortalecimiento de la comunicación entre personas adultas y niños y niñas. Al respecto, se coincide Maturana (2007), cuando plantea que los valores son aspectos relacionales espontáneos del convivir social que deben ser vividos en todo instante y no enseñados como nociones o acciones independientes.

\subsection{Percepción del equipo docente sobre el convivir y acciones que realizan en el aula para su promoción}

Para el grupo de maestras participantes en el estudio, la convivencia fue definida en términos de una vida en común, que implica compartir con otros, dar y recibir apoyo 
moral, espiritual y material, mantener comunicación y relaciones armónicas y pacificas con los demás. En lo que respecta al rol como protagonistas de la promoción de la convivencia en el escenario escolar, las acciones que ejecutan se sistematizan en la Tabla 7.

\section{Acciones que realizan docentes para promover convivencia en el aula}

Descripción

Actividades para demostrarse amor

Actividades que favorecen la comunicación

Actividades que promueven conocerse y compartir experiencias.

Practicar normas de cortesía y de respeto.

Enseñar límites y reglas en la clase

Actividades en equipo.

Actividades con enseñanzas
Ejemplos

Favorecer expresiones de afecto.

Orar juntos por un compañero enfermo

Escuchar a otro cuando habla sin interrumpir.

No permitir palabras ofensivas.

Expresar con respeto sentimientos de enojo.

Pensar antes de responder

Juegos organizados, dinámicas

Trabajos en subgrupos.

Saludar, dar gracias, pedir prestado, tolerar diferencias.

Esperar su turno, hacer fila, respeto por pertenencias ajenas, mantener limpio y ordenado su espacio, pedir permiso, seguir instrucciones, levantar la mano, respetar la autoridad de la maestra.

Tareas en el aula que impliquen ponerse de acuerdo, respetar otros puntos de vista, negociar.

Analizar situaciones de la vida diaria. Cuentos o historias con moralejas.

Como puede observarse en la tabla anterior, las profesoras se refirieron principalmente a actividades para favorecer la comunicación asertiva tales como: la escucha, el diálogo, esperar el turno para hablar, levantar la mano para hacerlo, el uso de lo que consideran el lenguaje apropiado, tono de voz, pensar antes de responder, así como evitar gestos y expresiones violentas, aspectos que coinciden con la resolución alternativa de conflictos y la adquisición de mayores habilidades sociales. 
Otras actividades propuestas enfatizaron en el valor del respeto: respeto a opiniones diversas, a la autoridad del docente, a las pertenencias ajenas. Las normas de cortesía también fueron mencionadas como enseñanzas de respeto. Compartir se presentó como un valor que practican en el aula, propiciando situaciones como juegos, dinámicas y proyectos, donde el estudiantado debe compartir materiales, ideas e incluso sentimientos.

El interés por el otro u otra (empatía) fue mencionado por el profesorado, mediante actividades que involucran la cooperación, la escucha, y la oración; a esto se suman las historias, cuentos y situaciones de la vida diaria para generar reflexión entre niños y niñas. Además, brindaron importancia al aprendizaje de reglas sociales para el convivir, como levantar la mano, seguir instrucciones, mantener limpieza y orden.

Es importante resaltar que cuando se cuestionó acerca de conductas y actividades concretas en pro del convivir en la escuela, la producción de las docentes participantes fue muy fructífera, lo que hace suponer que el rol que ejercen diariamente en el aula las ejercita en esta tarea. Sin embargo, sigue existiendo una distancia considerable entre realizar acciones específicas y aisladas, a lo que es un proyecto de vida orientado a la cultura de paz, donde se forme a las personas para ser conscientes de su papel protagónico en la construcción de ambientes sociales de convivencia.

\section{Conclusiones}

Aprender a convivir, de acuerdo con el Ministerio de Educación Chileno (2008) constituye la esencia de las relaciones sociales para la construcción de una sociedad más justa y una cultura de la paz, porque se sustenta en la dignidad de la persona, en el respeto y cuidado de sus derechos y sus deberes. Es un aprendizaje ligado a la formación de valores, al desarrollo de habilidades sociales, que se inicia en el espacio familiar y luego se traslada a los otros contextos

Las maestras participantes del estudio sustentaron una idea bastante clara acerca de la convivencia, en tanto comprenden que se trata de un proceso de aprendizaje en el que intervienen diversas instancias sociales, iniciando con el contexto familiar. El convivir tiene para ellas cimientos en la formación en valores, habilidades sociales y aprendizaje de normas modeladas congruentemente por figuras adultas.

Este dominio del tema puede explicarse desde el rol que cumplen como formadoras, algunas con experiencia de veinte años y más. Adicionalmente, por el contacto diario con una multiplicidad de estudiantes en que se tornan protagonistas y gestoras de la 
integración. Esto también explica que las maestras participantes conozcan y practiquen en la dinámica escolar una serie de estrategias para enseñar la convivencia.

En lo que respecta a los factores promotores e inhibidores del convivir entre las familias del estudiantado, las profesionales en educación tendieron a responder desde los antagonismos: valores-antivalores, disciplina (estilo de crianza democrático) -indisciplina (estilos de crianza disfuncionales), compartir tiempo en calidad y cantidad- tiempo limitado para compartir. A estos factores se sumaron otros secundarios, como la resolución de conflictos, el modelaje y la comunicación.

Las temáticas propuestas para capacitar y las sugerencias para educar la convivencia en el entorno familiar no fueron diferentes, sino que giraron en la misma sintonía de valores, disciplina, habilidades sociales, dedicar tiempo.

En términos generales, en las respuestas y reflexiones del equipo docente, prevalecieron las ejemplificaciones a través de experiencias cotidianas; también, con base en su sistema de creencias, que ellas comprenden como el "deber ser", muy ligado a la sumisión, la obediencia absoluta y, en algunos casos, a lo socialmente esperado ,según el género de pertenencia.

En estos planteamientos, las profesoras responsabilizaron de manera directa a las familias, especialmente a los padres y las madres, que de acuerdo con ellas no cumplen con funciones asignadas para la crianza. Al respecto, López (2005) recuerda que en las últimas décadas la familia le ha delegado este aprendizaje a la escuela, que es vivido por las maestras como un recargo de labores. Adicionalmente, opinaron que esta omisión, por parte de la instancia socializadora primaria, repercute en que niños y niñas establezcan relaciones violentas y las reproduzcan en el escenario escolar.

Es importante destacar que la denuncia de las participantes se dirigió de manera exclusiva al entorno familiar, que no fue contemplado como un escenario de menor envergadura, inserto en un contexto, social, cultural y político que dicta las pautas a seguir a las diferentes instancias sociales.

Para las Naciones Unidas (1998) implicar a las familias en la educación pacificadora requiere desarrollar formas de pensamiento crítico ante la sociedad; reflexionar y asumir valores, normas y actitudes facilitadoras de la convivencia y capaces de dar respuesta a los problemas que se les plantean diariamente; reconocer y aceptar los valores que existen en la diversidad de los individuos, los sexos, los pueblos y las culturas y desarrollar la capacidad de comunicar, compartir y cooperar con los demás. 
Contrariamente, el discurso del grupo de las maestras se enfocó en señalar manifestaciones conductuales y acciones concretas, no incorporó elementos de índole cognitiva, de replanteamiento de los esquemas mentales, de incorporar otros paradigmas para la interpretación de la realidad, y/o de la posibilidad de generar discusiones y pensamiento reflexivo. Los niños y las niñas no fueron percibidos como protagonistas, sino como entes pasivos sujetos al modelaje de las figuras adultas, con reducidas posibilidades de cambio.

Al respecto, plantea Garnier (2008) que enseñar y aprender a convivir con otros en forma respetuosa, solidaria y democrática requiere vencer múltiples desafíos mundiales. Entre ellos nuevos marcos para interpretar la realidad, diferentes actitudes y prácticas y una coherencia entre el discurso y el funcionamiento real. Además, requiere crear conciencia de la necesidad de cambiar formas de relación y comunicación, estilos de poder y autoridad.

Finalmente, se debe tener presente que la convivencia es un compromiso colectivo que implica un proyecto como sociedad. Es a la vez un desafío y un aprendizaje, un tema que nos compete a todos y todas $y$, por lo tanto, imprescindible fortalecer en todas las esferas involucradas en su promoción.

\section{Referencias}

Ares, Patricia (2002). Psicología de Familia. Una aproximación a su estudio. La Habana, Cuba: Editorial Félix Varela.

Brunner, Jerome. (1990). La Autobiografía del Yo. En: Actos de Significado. Más allá de la revolución cognitiva (pp. 101-133). Madrid: Alianza Editorial.

Chile, Ministerio de Educación. (2002). Política de Convivencia Escolar hacia una educación de calidad para todos. Santiago, Chile: MINEDUC.

Chile, Ministerio de Educación. (2008). Estudio Nacional de Convivencia Escolar. Santiago, Chile: MINEDUC.

Colombia, Ministerio de Educación Nacional. (2005). Política Educativa para la formación escolar en la convivencia. Recuperado de www.mineducacion.gov.co/1621/article90103.html

Edwards, Luis. (2002). Políticas de Convivencia Escolar: hacia una educación de calidad para todos. Chile: MINEDUC

Espinosa, María. (2009). Panorama de las relaciones familiares en la época actual. En Marco Murueta y Maricela Osorio (Eds.), Psicología de la familia en países latinos 
del siglo XXI (pp. 43-60). Recuperado de http://newpsi.bvspsi.org.br/ebooks2010/pt/Acervo files/PsicologiaFamiliaLatinosXXI.pdf

Garnier, Leonardo. (2008). Conferencia inaugural en III Jornadas de Cooperación Iberoamericana: educación para la paz, la convivencia democrática y los derechos humanos. San José, C.R.: IIDH.

Geertz, Clifford. (1994). Conocimiento Local: Ensayo sobre la interpretación de las culturas. Buenos Aires: Editorial Paidós.

Hernández, Roberto, Fernández, Carlos, y Baptista, Pilar. (2006). Metodología de la investigación. México, D.F.: McGraw Hill.

López, Jesús. (2005). Proyecto Educativo para la mejora de la convivencia. Revista Currículum Formación del Profesorado. Recuperado de http://www.ugr.es/ recfpro/rev91COL3.pdf

Maturana, Humberto. (2007). Transformación en la convivencia. Chile: Comunicaciones Noreste LTDA.

Muñoz, María, Saavedra, Eugenio y Villalta, Marco. (2007). Percepciones y significados sobre la convivencia y violencia escolar. Revista de Pedagogía, 28(82), 197-224.

Murueta, Marco. (2009). Familia y proyecto social en la teoría de la praxis. En Marco Murueta y Maricela Osorio (Eds.), Psicología de la familia en países latinos del siglo $X X I \quad$ (pp. 23-41). Recuperado de http://newpsi.bvspsi.org.br/ebooks2010/pt/Acervo files/PsicologiaFamiliaLatinosXXI.pdf

Naciones Unidas. (1998). Carta de las Naciones Unidas y Estatuto de la Corte Internacional de Justicia. Recuperado de http://www.un.org/es/documents/charter/

Rodríguez, Bertha. (2006). Los métodos alternativos de solución de conflictos: una estrategia inteligente para facilitar la convivencia pacífica. Revista Trabajo Social, 3, 124-139.

Tuvilla, José. (2004). Cultura de Paz. España: Editorial Descléer de Brouwer, S.A.

UNESCO. (2007). Educación de calidad para todos: un asunto de Derechos Humanos. Santiago, Chile: Salesianos Impresores S.A.

UNESCO. (2008). Convivencia democrática, inclusión y cultura de paz. Santiago, Chile: Salesianos Impresores S.A.

Villarroel, Gladys y Sánchez, Ximena. (2002). Relación familia y escuela: un estudio comparativo en la ruralidad. Revista Estudios Pedagógicos, (28), 123-141. 